doi:10.17659/01.2020.0058

Journal of Case Reports 2020;10(4):223-225

\title{
Tuberous Sclerosis: A Diagnostic Clinical Dilemma: Review and Case Report
}

\author{
Priti Singh ${ }^{1}$, V. Chandar ${ }^{2}$
}

${ }^{1}$ Department of Pediatrics, Dr. Ram Manohar Lohia Hospital and Post Graduate Institute of Medical Education and Research, New Delhi; ${ }^{2}$ Department of Pediatrics and Neonatology, Swami Rama Himalayan Institute of Medical Sciences, Jolly Grant, Dehradun, Uttarakhand, India.

\section{Corresponding Author:}

Dr Priti Singh

Email: drpritisingh1532@gmail.com

This is an Open Access article distributed under the terms of the Creative Commons Attribution License (creativecommons.org/ licenses/by/3.0).

Received

Accepted

Published
June 29, 2020

: August 16, 2020

October 25, 2020

\begin{abstract}
Background: Tuberous sclerosis is neuro-cutaneous, multisystem disease affecting children and adults, characterized by the formation of hamartomatous lesions in multiple organs like brain, kidney, lung, heart and skeleton system. Case Report: We hereby report a 10 year old who presented in hypertensive encephalopathy making tuberous sclerosis a diagnostic dilemma. The investigations revealed cutaneous involvement, intracranial lesion and renal lesion. Conclusion: These patients require multidisciplinary support and prolonged palliative measures for better outcome and survival.
\end{abstract}

Keywords: Angiomyolipoma, Blood Pressure, Scabies, Seizures, Tuberous Sclerosis.

\section{Introduction}

Tuberous sclerosis (TS) is an autosomal dominant, neurocutaneous, multisystem disease affecting children and adults, characterized by the formation of hamartomatous lesions in multiple organs like brain, kidney, lung, heart and skeleton system with a birth incidence of around one in 10,000 [1]. The disease results from mutations in one of two genes, TSC1 (encoding hamartin on chromosome 9q3234 or TSC2 (encoding tuberin on chromosome 16p13.3), which have an important role in the regulation of cell proliferation and differentiation [2].

The disease causes disabling neurological disorders including epilepsy, mental retardation and autism. The other major features of the diseases are dermatological manifestations like renal angiomyolipoma (AML), facial angiofibroma, and pulmonary lymphangiomatosis. It has a wide spectrum of presentation with no neurological symptoms to severe epilepsy, encephalopathy and lung manifestation.

\section{Case Report}

A 10 year old male child presented to our emergency department with history of fever for one day and repeated convulsions in last 24 hours. His past history was suggestive of one episode of convulsion 2 years back. There was no history of trauma, epilepsy, and contact with tubercular patient. The child was born at full term with normal delivery and had normal growth. The family history was not contributory. On physical examination, the child was unconscious and in respiratory distress with respiratory rate: $36 /$ minute, heart rate: $123 /$ minute, temperature $102^{\circ} \mathrm{F}$ and blood pressure 160/136 mmHg. Systemic examination revealed adenoma sebacum present on face and eye lids [Fig.1a], Asch leaf scar on forehead, Shagreen patch on lower back, periungal fibroma on right foot [Fig.1b], hyper-pigmented macules present all over the abdomen and scabies lesion in the interdigital cleft of fingers. Neurological examination did not reveal any sign of meningeal irritation with $3 / 4$ power in all four limbs and bilateral plantar flexor response. Rest of the systemic examination 
did not show any positive findings. Hematological investigations did no yield any positive finding to make a provisional diagnosis.

Multiple hyperechoic lesions were seen in cortical region of both the kidneys with minimal free fluid in peritoneal cavity on ultrasound suggestive of renal angiomyolipoma. MRI brain showed multiple sub-ependymal nodules with multiple cortical tubers [Fig.2a,b]. No abnormality was seen in $\mathrm{x}$-ray chest and echocardiography. The patient was put on anti-hypertensive, antibiotics, steroids, anti-epileptic and ICT reducing drugs and supportive measures. He improved and was discharged after a week of treatment.

\section{Discussion}

Tuberous sclerosis (TS), also known as tuberous sclerosis complex or Bourneville disease, is a neurocutaneous disorder characterized by development of multiple benign tumors of the embryonic ectoderm (e.g. skin, eyes, and nervous system). The clinical triad of papular facial nevus, seizures and mental retardation is found in less than half of the patients. The radiological hallmarks of this neurocutaneous syndrome are universally accepted as sufficient for diagnosis [3]. Tuberous sclerosis was classically described as presenting in childhood with a triad (Vogt triad) of seizures (absent in one-quarter of individuals), mental retardation (up to half have normal intelligence), adenoma sebaceum (only present in about three-quarters of patients) [4]. The full triad is only seen in a minority of patients $(30 \%)$. Therefore, TS has a wide clinical spectrum. The diagnosis of definitive TS is based on specific clinical features and requires the presence of two major criteria or one major and two minor criteria [5]. Pulmonary lymphangioleiomyomatosis, renal angiomyolipoma and facial angiofibroma are some of the major clinical features. The most frequent cause of death in patients with TS is renal complication [6].

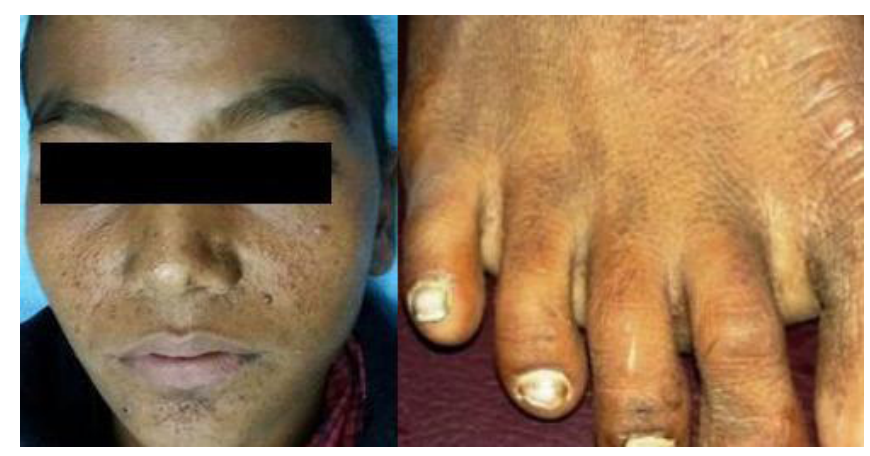

Fig.1: Adenoma sebacum on face and eye lids and periungal fibroma on foot.

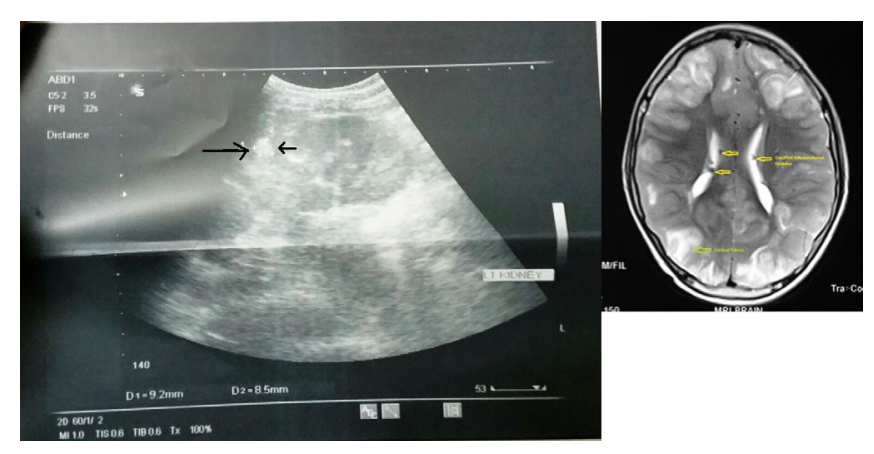

Fig.2: Ultrasound showing renal angiolipoma and MRI brain showing cortical tuber and calcified subependymal nodules.

Classical central nervous system involvement includes cortical tubers, subependymal nodules, sub-ependymal giant cell astrocytoma and benign white matter lesions. Tubers are benign lesions composed of dysmorphic neurons occupying a cortical or sub-cortical location. By age ten, fifty percent of patients have calcified cortical tubers [7]. Subependymal nodules are found in nearly ninety-five percent of patients with tuberous sclerosis. The commonest site is near the caudate nucleus along the striothalamic groove of the lateral ventricle.

Renal lesions in tuberous sclerosis commonly consist of simple renal cyst and angiomyolipomas. Angiomyolipomas are benign in nature. They may arise in renal cortex or medulla. They are usually multiple and bilateral and are present in $50-80 \%$ of patients with 
tuberous sclerosis [8]. On sonography classically angiomyolipomas are echogenic relative to renal parenchyma. If vascular element predominates, they may be hypoechoic. Renal cell carcinoma though rarely associated with tuberous sclerosis, is one of the significant manifestations $[9,10]$.

Cutaneous lesions are present in 95\% of patients, these include facial angiofibroma (adenoma sebaceum), subungal fibromas, and shagreen patches. Skeletal involvement is present in $50 \%$ of patients and is typically asymptomatic. Common findings include bone islands in diploic spaces of skull vault and in pelvic bones, cysts and undulating periosteal reaction in metatarsals, metacarpals and phalanges. In pelvis, iliac blades are sites of flame shaped densities.

Pulmonary findings are seen in less than $1 \%$ of patients with tuberous sclerosis. They occur in form of cystic lymphangiomyomas, and chronic fibrosis. Cardiac rhabdomyomas are true hamartomas found in approximately 30\% patients with tuberous sclerosis, predominantly children. Retinal hamartomas may occur in some patients but usually do not impair vision. Vascular abnormalities occur in form of aneurysms of thoracic or abdominal aorta. Vascular dysplasia with progressive occlusion of craniocervical vessels has been reported in association with moyamoya like pattern of collateral circulation. Liver leiomyomas or adenomas may rarely occur.

\section{Conclusion}

Our patient presented in a state of hypertensive encephalopathy making a diagnostic dilemma and investigations revealed cutaneous involvement, intracranial lesion and renal lesion. Though benign lesions, yet may affect the functioning of various systems indirectly. These patients require multidisciplinary support and prolonged palliative measures for better outcome and survival.

Contributors: PS: manuscript writing and editing, patient management; VC: manuscript writing and literature review. PS will act as a study guarantor. Both authors approved the final version of this manuscript and are responsible for all aspects of the study.

Funding: None; Competing interests: None stated.

\section{References}

1. Hancock E, Tomkins S, Sampson J, Osborne J. Lymphangioleiomyomatosis and tuberous sclerosis. Respir Med. 2002;96(1):7-13.

2. Crino PB, Nathanson KL, Henske EP. The tuberous sclerosis complex. N Engl J Med. 2006;355:1345-1356.

3. Braffman BH, Bilaniuk CT, Zimmerman RA. MR of central nervous system neoplasia of the phakomatoses, Sem Roentgenol. 1990;25:198-217.

4. Umeoka S, Koyama T, Miki Y, Akai M, Tsutsui K, Togashi K. Pictorial review of tuberous sclerosis in various organs. Radiographics. 28 (7):e32. doi:10.1148/ rg.e32.

5. Curatolo P, Bombardieri R, Jozwiak S. Tuberous sclerosis. Lancet. 2008;372:657-668.

6. Tuzel E, Kirkali Z, Mungan U, Culer C, Sade M. Giant angiomyolipoma associated with marked pulmonary lesions suggesting lymphangioleiomyomatosis in a patient with tuberous sclerosis. Int Urol Nephrol. 2000;32:219-222.

7. Kingsley D, Kendall B, Fitz C. Tuberous sclerosis: a clinicoradiological evaluation of 110 cases with particular reference to atypical presentation. Neuroradio. 1986;28:171-190.

8. Stillwell TJ, Gomez MR, Kelalis PP. Renal lesions in tuberous sclerosis. J Urol. 1987; 138:477-481.

9. Lendvay TS, Broecker B, Smith EA. Renal cell carcinoma in a two year old child with tuberous sclerosis. J Urol. 2002;168(3):1131-1132.

10. Kulkarni S, Uddar M, Deshpande SG, Vaid S, Wadia RS. Renal cell carcinoma as significant manifestation of tuberous sclerosis complex. J Assoc Physicians India. 2000; 48(3):351-353. 